J. Acoust. Soc. Jpn. (E) 9, 6 (1988)

\section{A statistical characteristic of a physical measure of "proficient speech"}

PACS number: 43.70.Fq, 43.71. Es, 43.71.Ar

I. Kitagaki

The Research and Development Institute of Vocational Training,

Employment Promotion Corporation, 1960 Aihara, Sagamihara, 229 Japan

(Received 26 March 1988)

\section{Introduction}

Concerning the proficiency of a person's speech, it is very probable that any impression depends upon some acoustic information. We here extract a statistical characteristic of the length of the segment which means the length of a passage spoken without pauses, then suggest a significant factor as to the proficiency of speech.

The author has worked on the characterization of a teacher's speaking skill based upon the acoustic information. ${ }^{1)}$ As an unexpected data distribution was observed by chance during the work, this data and its interpretation are also reported.2)

2. Measurement of the Length of the Speech Segment The classroom proceedings of several teachers were individually recorded in an audio-digital way. There were six members, one of them, so to speak, an experienced teacher and the others trainees (student teachers). Each proceeding of several minutes was listened to by subjects (seventy-five college students) who evaluated the proficiency of each speech using an answering sheet. The questionnaires consisted of six items all of which were concerned with phonetic features including "distinctive intonations," "speaking with pauses and intonations," and "not regimental." The answering sheet was designed so that each item formed an ordinal scale; the subjects checked a number from one through five. One and five corresponded respectively to the lowest and the highest proficiency. When listening to the records, the subjects were not informed which was the experienced teacher's.

The scores were averaged both over all subjects and the six items. The averaged scores ranged from 2.3 to 3.1 in the case of the trainees; on the other hand, the highest score among the seven experienced teachers was 3.8. This highest scoring teacher is hereafter referred to as $S_{\text {sup. }}$.
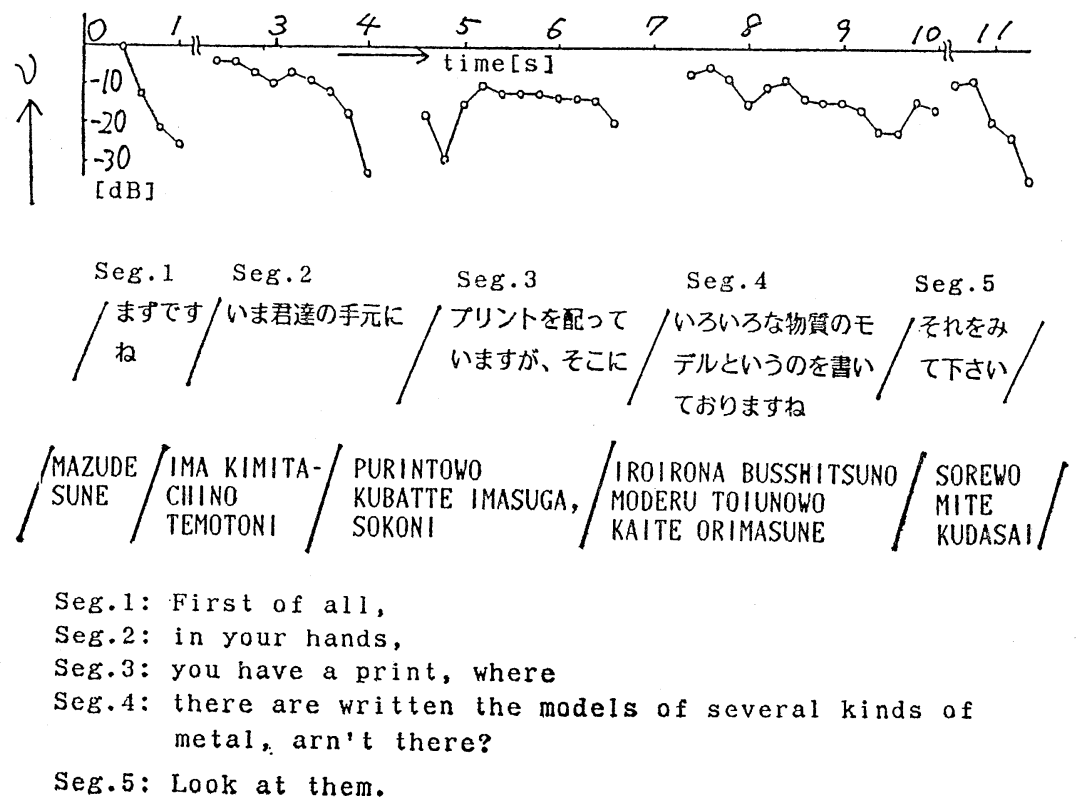

Fig. 1 Sound pressure level $v$ in a teacher's speech. 
The physical measurement was done for two speakers,

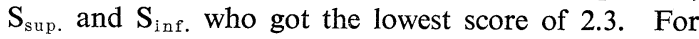
each data, the segment was measured for about two minutes.

Figure 1 shows the sound pressure level for each of five spoken segments of speaker $S_{\text {sup. }}$. Sound prssure level $v$ is adjusted so that the maximum value in the analyzed period is to be $0 \mathrm{~dB}$. Each segment refers to the period sandwiched between two pauses adjacent to each other, neither of which was caused by a double consonant. In this case a pause was defined as the part where sound pressure level $v$ is lower than a certain value of $v_{t}$. Here, $v_{t}$ was set to be $-35 \mathrm{~dB}$. So, a segment can be said to be "a passage spoken without pauses." In the figure, the part between two slashes is one segment.

The length $l \mathrm{~s}$ of a segment was measured by observing the envelope on an $x-t$ recorder with an accuracy of $\pm 0.1 \mathrm{~s}$. The envelope of a segment is shown in Fig. 2. In this figure, the starting point of the segment is located just ahead of the first convex curve, $6 \mathrm{~dB}$ lower than the maximum value. The location of the ending point is determined almost in the same way as the starting point.

Figure 3 shows the normalized frequency of occurrence of the length of the segments. The total numbers of the segments were thirty and thirty-seven for speakers $S_{\text {sup. }}$ and $S_{\text {inf., }}$ respectively. Those figures were obtained as follows, considering the error of measurement. Given a measured length of $l_{0}$, we convert it to a possibility function $f$ shown in Fig. 4:

$$
\begin{array}{ll}
f=1 & \text { for } l_{\mathrm{L}} \leq l_{0}<l_{\mathrm{U}} \\
f=0 & \text { otherwise }
\end{array}
$$

where $l_{0}-l_{\mathrm{L}}=0.1$ and $l_{\mathrm{U}}-l_{0}=0.1$ hold good. All data were processed in the same way. And accumulating all data, the distribution $F$ was obtained. Then, we got Fig. 3 through normalizing the distribution by the maximum value $F_{\max }$.

A remarkable difference is observed comparing Fig. (a) with Fig. (b). Figure (a) is characterized by bimodal distribution. In the detail, the distribution is concentrated at $1 \mathrm{~s}$ and at a little longer than $2 \mathrm{~s}$. On the other hand, this tendency of the bimodal is not observed in Fig. (b).

Incidentally, we assess the bimodal distribution by the areas in the figures. Given the designation of areas A, B and the total area in Fig. (a) as $P_{\mathrm{A}}, P_{\mathrm{B}}$ and $P_{\text {sup. }}$, respectively, the following is obtained:

$$
\begin{aligned}
& P_{\mathrm{A}} / P_{\text {sup. }}=0.47 \\
& P_{\mathrm{B}} / P_{\text {sup. }}=0.37
\end{aligned}
$$

Because the sum of these two areas is more than eighty percent of the total, it is specified that bimodal distribution is the remarkable characteristic of the figure. In other words, the speech of speaker $S_{\text {sup. }}$ is statistically marked by two essential characteristics.

On the other hand, in Fig. 3(b), area C includes around eighty percent of the total. Using the similar

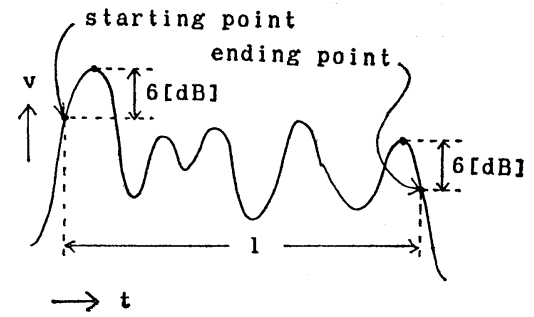

Fig. 2 Envelope of a segment.

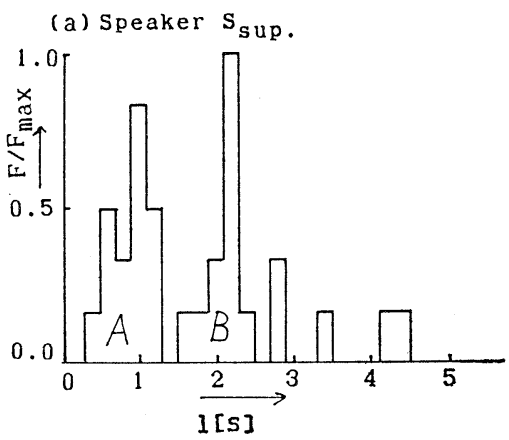

(b) Speaker $s_{\text {inf }}$.

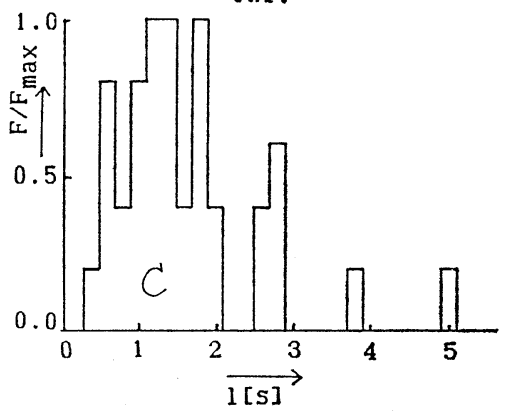

Fig. 3 Normalized distribution of the length of segments.

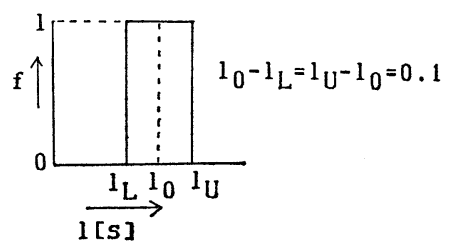

Fig. 4 Possibility function.

designation, we discover that $P_{\mathrm{C}} / P_{\text {inf. }}$ is equal to 0.79 .

3. Conclusion

Although this study is based upon a limited data, a 


\section{KITAGAKI: STATISTICAL CHARACTERISTIC PHYSICAL MEASURE PROFICIENT}

factor concerning "proficient speech" is suggested. It may extend to basic study of essential characteristics in man-to-man communication, in speech synthesis aiming at natural colloquial language, and so forth. The data distribution shown here emphasizes the necessity for further research in order to identify whether or not the bimodal distribution above is essential in proficient speech.

\section{References}

1) I. Kitagaki and K. Shindo, "Evaluating teacher's speaking skills and vocal characteristics," Jpn. J. Educ. Technol. 10(3), 19-29 (1986) (in Japanese).

2) I. Kitagaki, "On the existence of "rhythm in speech," Trans. Inst. Electron. Inf. Commun. Eng. J70-A, 846-847 (1987) (in Japanese). 